\section{NP17 Publication Patterns of PhD Students in the Illinois Transdisciplinary Obesity Prevention Program Versus Traditional Programs}

Sharon Donovan, PhD, RD, sdonovan@illinois.edu, Department of Food Science \& Human Nutrition, University of Illinois, 339 Bevier Hall, 905 South Goodwin Avenue, Urbana, IL 61801; B. Fiese, PhD; J. Liechty, PhD; A. S. Keck, PhD, CCRP

Objective: To compare publication patterns of doctoral students in a transdisciplinary research-based $\mathrm{PhD} / \mathrm{MPH}$ degree program (I-TOPP) focused on child obesity prevention to students enrolled in a traditional $\mathrm{PhD}$ program within the same academic units.

Description: Publication records of I-TOPP $(n=11)$ and traditional $\mathrm{PhD}(\mathrm{n}=29)$ students were tracked from matched time of enrollment 2011-2013 to January 2016.

Evaluation: Number of peer-reviewed publications, year of publication, number of authors and departments per publication, and number of citations on Google Scholar were compared between I-TOPP and traditional students. Statistical differences were determined by t-test, $\mathrm{p}<0.05$.

Conclusion and Implications: The I-TOPP students produced $4.5 \pm 2.9$ publications per student (49 total in 38 journals) compared to $2.1 \pm 0.6$ publications per student (61 total in 51 journals) produced by the traditional doctoral students, but this difference was not significant. Compared to traditional PhD students, I-TOPP students had more co-authors per publication $(7.0 \pm 0.4$ vs. $5.3 \pm$ $0.4, \mathrm{p}=0.003)$, more departments represented per publication ( $3.4 \pm 0.2$ vs. $2.4 \pm 0.2, \mathrm{p}=0.002)$ and more organizations on each publication $(2.35 \pm 0.22$ vs. $1.7 \pm 0.1$, $\mathrm{p}<0.001)$. To assess impact, the number of citations in Google Scholar was determined. The number of citations was 4-fold higher $(\mathrm{p}<0.001)$ per publication for I-TOPP than traditional $\mathrm{PhD}$ students $(16.2 \pm 3.7$ vs. $3.1 \pm 0.7)$. Findings suggest that transdisciplinary training does not negatively impact student publication records. Trandisciplinary trained scholars had publications with more diverse collaborations and higher impact than those from students in traditional doctoral programs.

Funding: USDA Grant \#2011-67001-30101

\section{NP18 Engaging Youth as Advocates to Create Healthy Snacking Zones Around Rural Schools - Year 4}

Nancy Findholt, PhD, RN, findholt@ohsu.edu, Oregon Health \& Science University, One University Boulevard, La Grande, OR 97850; B. Izumi, PhD, MPH, RD, Portland State University; J. Shannon, $P h D, R D, M P H$;

T. Nguyen, MD, PhD; C. Smith, MEd

Objective: This on-going 4-year quasi-experimental study is evaluating the effects of an intervention that engages 4-H youth participants in advocacy for "healthy snacking zones" within 5 elementary/middle schools and nearby small food stores in a rural Oregon county.
Description: Youth have received nutrition education, participated in a photovoice assessment to build awareness of environmental barriers to healthy snacking and, with support from project staff and adult volunteers, have planned and implemented several outreach projects to promote healthy snacking within their schools and communities. Currently, club members are working with food store owners to increase availability and promotion of healthy snacks and beverages in stores near their schools.

Evaluation: Mixed methods are being used to evaluate intervention implementation, environmental change, and changes in children's snacking behaviors and BMI.

Conclusions and Implications: Preliminary results show a significant intervention effect on teachers' nutrition knowledge relevant to snacks $(\mathrm{p}=0.054)$, and decreased use of candy as rewards/incentives for students $(\mathrm{p}=0.005)$. Likewise, there have been improvements in students' snacking behavior, including statistically significant decreases in consumption of regular chips and sweetened fruit drinks (Cohen's $\mathrm{d}=0.06$ and 0.03 , respectively), and six food stores have designated shelving and refrigerator space that is stocked exclusively with foods and beverages that meet USDA Smart Snacks nutrition criteria. In conclusion, preliminary results suggest that youth advocacy is an effective strategy for promoting healthy snacking within rural schools and communities.

Funding: USDA Grant \#2012-68001-19702

\section{NP19 Optimizing a Childhood Obesity Prevention Program Using an Engineering- Inspired Framework}

Lori Francis, PhD, lfrancis@psu.edu, Pennsylvania State University, 219 Biobehavioral Health Building, University Park, PA 16802; R. BeLue, PhD; K. Kugler, PhD

Objective: Effective programs to prevent obesity among preschool children have been largely unsuccessful. Using a novel approach to optimizing behavioral interventions, the multiphase optimization strategy (MOST), we will examine the effects of four intervention strategies over the course of 12 weeks on modifying the classroom environment in center-based childcare settings to increase vegetable intake, active play, and improve self-regulatory capacity in preschool children.

Description: MOST is an engineering-inspired framework that facilitates the selection of efficacious intervention components by first identifying and piloting the components, then assessing their individual and combined effects through efficient randomized experimentation. Following the principles of MOST we will identify one of the best combinations of intervention components in a thoughtful and systematic way. This is in contrast to the classical approach to intervention development with the 2-arm RCT, where even if the intervention is deemed efficacious, we would not know which components were efficacious and which were unnecessary. Thus using the principled framework of MOST, we increase the likelihood 


\section{NP19 (continued)}

of building a more efficient and effective childhood obesity prevention program.

Evaluation: Using a factorial study design, we will examine the efficacy of 4 components: classroom curriculum designed to increase children's nutrition knowledge, increase children's active play and to improve children's emotional, behavioral and energy intake regulation, in addition to a parenting component that reinforces the messages outlined in the classroom curricula by providing guidance on ways to increase/improve child outcomes. Evaluation plans will be outlined.

Conclusions and Implications: Testing the efficacy of each intervention component separately and together will allow us to design the most efficient, efficacious and cost-effective intervention.

Funding: USDA Grant \#2015-68001-23233

\section{NP20 Process Evaluation of the Smarter Lunchrooms Randomized Controlled Trial: Years 1-3}

Alisha Gaines, PhD, CHES, againes@cornell.edu, Cornell University, Division of Nutritional Sciences, 342A Martha Van Rensselaer Hall, Ithaca, NY 14853; A. Gaines, PhD;

T. F. Hill, MPH; L. N. Thomas, PhD; D. R. Just, PhD;

B. Wansink, PhD; J. S. Dollahite, PhD

Objective: To conduct a process evaluation of the Smarter Lunchrooms randomized controlled trial based in middle school cafeterias and designed to improve students' fruit, vegetable, and unflavored milk selection and consumption through small, low cost environmental changes.

Description: Throughout the 2013-2015 school years, fruit, vegetable, and milk protocols were tested separately and then in combination in a total of 24 public middle schools in New York State. Campus and school staff worked with local Cooperative Extension agents to implement changes. An adapted RE-AIM framework was used to monitor protocol fidelity, determine protocol maintenance post-intervention, and identify facilitators and barriers to implementation.

Evaluation: Pre-, mid-, and post-intervention cafeteria audits were used to generate semi-quantitative measures of protocol implementation and maintenance. Training materials and associated evaluations, environmental assessments, and semi-structured interviews with Cooperative Extension agents, food service directors, and cafeteria managers were analyzed using qualitative techniques to identify themes related to facilitators, barriers, and opportunities for improving intervention delivery.

Conclusions and Implications: Analyses revealed varied fidelity to and limited maintenance of intervention protocols. Progression records and evaluations indicated consistent satisfaction with trainings provided to Cooperative Extension and school staff. Implementation challenges were typically related to limitations of cafeteria structures or school staff motivation. These data high- lighted targets for intervention redesign, for example, enhanced communication among all participating staff and increased efforts to fostering school staff buy-in. Findings will also strengthen interpretation of outcome data. Results will be useful for supporting nationwide Smarter Lunchroom efforts and should offer insight for other school-based environmental interventions.

Funding: USDA Grant \#2012-68001-19604

\section{NP21 Development of a Mindful Eating Intervention Among Elementary School Children and Their Parents}

Keiko Goto,PhD, kgoto@csuchico.edu, California State University, Chico, 400 West 1st Street, Chico, CA 95929; C. Wolff, PhD, RD, MPA; J. Giampaoli, PhD, RD; B. Seipel, PhD; A. Wylie, BA; S. Pierson, BA

Objective: The current study examined the feasibility and appropriateness of proposed activities for a mindful eating intervention among third through fifth grade children and their parents in northern California.

Description: Four focus groups and a parent workshop were conducted with parents of children from a lowincome elementary school.

Evaluation: Study results revealed that parents valued mindful eating practices and demonstrated openness to learning mindful food parenting skills. Parents also showed a desire to obtain simple and healthy recipes and involve their children in mindful cooking at home.

Conclusions and Implications: Six monthly mindful eating lessons and activities for classroom implementation, as well as mindful eating activities for use by parents and children at home, were developed and evaluated by a professional panel. Mindful eating practice topics include: enjoying sensory aspects of food, hunger and fullness awareness, emotional awareness, limiting environmental distractions that promote mindless eating, identifying resources and individuals involved in producing food, and preparing and eating cultural dishes together as a family.

Funding: USDA Grant \#2015-69001-23238

\section{NP22 Smarter Lunchrooms Randomized Control Trial: Results From Year 3}

Katherine Greene, MPH, kng29@cornell.edu, Cornell University, 475 Warren Hall, Ithaca, NY 14853; G. Gabrielyan, PhD; A. Brumberg,; J. Dollahite, PhD; D. R. Just, PhD; B. Wansink, PhD

Objective: In Year 3, the objective was to determine the impact of combining multiple Smarter Lunchroom interventions on the selection and consumption of healthy food items in the school lunchroom. This design was informed by the positive results of single Smarter Lunchroom protocols in Year 2.

Description: In partnership with Cornell Cooperative Extension, 11 middle schools from urban and rural 